\title{
Control of rhodopsin activity in vision
}

\section{Abstract}

Although rhodopsin's role in activating the phototransduction cascade is well known, the processes that deactivate rhodopsin, and thus the rest of the cascade, are less well understood. At least three proteins appear to play a role: rhodopsin kinase, arrestin and recoverin. Here we review recent physiological studies of the molecular mechanisms of rhodopsin deactivation. The approach was to monitor the light responses of individual mouse rods in which rhodopsin was altered or arrestin was deleted by transgenic techniques. Removal of rhodopsin's carboxy-terminal residues which contain phosphorylation sites implicated in deactivation, prolonged the flash response 20 -fold and caused it to become highly variable. In rods that did not express arrestin the flash response recovered partially, but final recovery was slowed over 100 -fold. These results are consistent with the notion that phosphorylation initiates rhodopsin deactivation and that arrestin binding completes the process. The stationary night blindness of Oguchi disease, associated with null mutations in the genes for arrestin or rhodopsin kinase, presumably results from impaired rhodopsin deactivation, like that revealed by the experiments on transgenic animals.

Key words Arrestin, Kinase, Night blindness, Rhodopsin, Rod, Transgenic

Rhodopsin's role in vision is well known; upon absorption of light it activates a G-protein cascade that generates an electrical response at the surface membrane of the retinal rod cell. This response encodes the absorption of single photons, and upon transfer through the visual pathway it ultimately elicits visual sensations. Rhodopsin's role in signal generation within the rod is diagrammed in Fig. 1.

Photoisomerised rhodopsin catalyses GTP/ GDP exchange on the G-protein transducin, a single rhodopsin producing hundreds of copies of activated transducin $\left(\mathrm{T}_{\mathrm{GTP}}\right)$ within a fraction of a second. $\mathrm{T}_{\mathrm{GTP}}$ activates a cyclic GMP phosphodiesterase (PDE). This enzyme hydrolyses cyclic GMP (cGMP), a diffusible internal messenger that is present at relatively high concentration in the cytoplasm in darkness and that binds to cationic channels in the surface membrane, holding them open. Hydrolysis of cGMP allows the channels to close, interrupting an inward current of sodium, calcium and magnesium ions and producing a hyperpolarisation of the membrane. The hyperpolarisation reduces the rate at which neurotransmitter is released from the synaptic terminal of the rod.

The purpose of this paper is to review recent work on the important but still poorly understood mechanisms that terminate the light-evoked catalytic activity of rhodopsin. These mechanisms, which fix the intensity and duration of the activation of the transduction cascade, need to satisfy strong functional constraints. Rhodopsin activity must be terminated rapidly so that an absorbed photon can be registered without contamination by after-effects of previous absorptions, yet it must last long enough to give an easily detectable electrical response. Furthermore, activity should be shut off in a stereotyped fashion so that each absorbed photon is encoded identically, allowing the brain to make an accurate assessment of the number and timing of photon absorptions. Finally, shut-off should be complete and irreversible, since escape of recently deactivated rhodopsin molecules to the active state may falsely signal photon absorptions, generating masking noise that interferes with normal vision.

Given these constraints, it is perhaps not surprising that the deactivation of rhodopsin seems to involve fairly complex mechanisms. Our current understanding of the process, drawn largely from in vitro biochemical experiments, is summarised in Fig. 2. At least three proteins seem to play key roles: rhodopsin kinase, arrestin/p44 and recoverin.

Photoactivated rhodopsin can be rapidly phosphorylated at multiple serine and threonine residues near its carboxy(C)terminus. ${ }^{1}$ This phosphorylation is catalysed by rhodopsin kinase. ${ }^{2}$ Phosphorylation of rhodopsin reduces its ability to activate transducin and permits the binding of the soluble capping protein arrestin, which further quenches rhodopsin's activity. ${ }^{3,4}$ Recoverin, a calcium-binding protein, can inhibit rhodopsin phosphorylation in vitro ${ }^{5}$ by binding to
D.A. Baylor

M.E. Burns

Department of

Neurobiology

Stanford University School

of Medicine

Stanford

CA94305, USA

Tel: +1 (650) 723-6510

Fax: $+1(650) 725-3958$

This research was supported by Grant EY05750 from the National Eye Institute, the Ruth and Milton Steinbach Fund, and the McKnight Foundation 


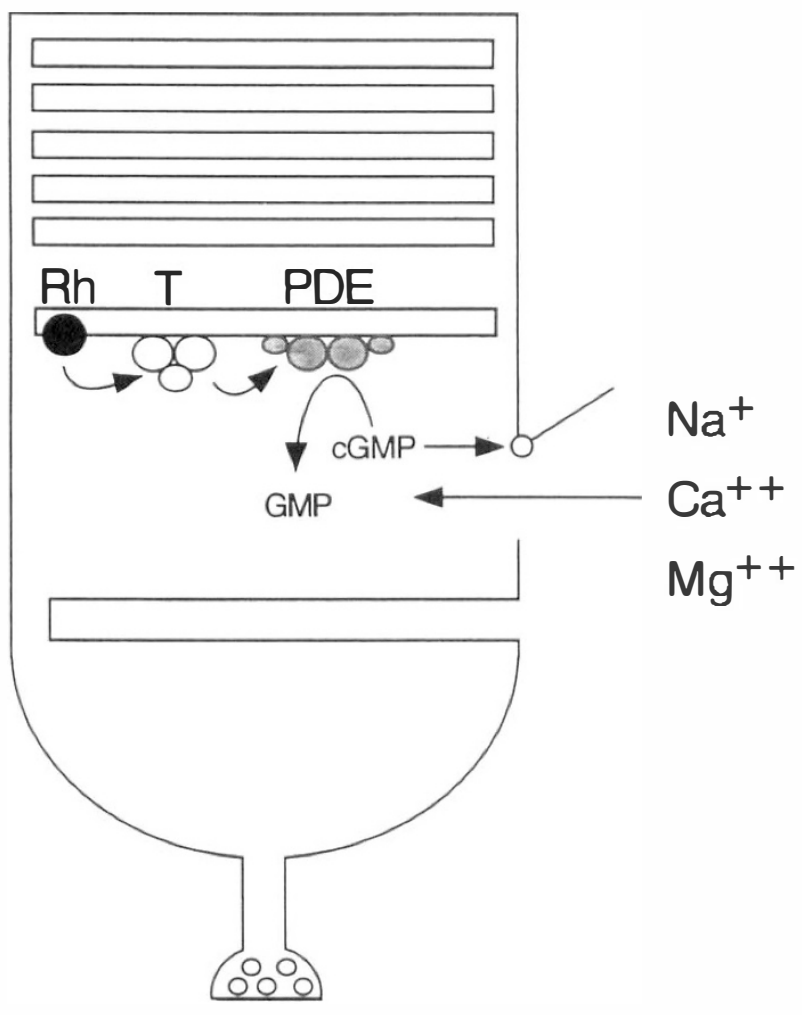

Fig. 1. Amplifying cascade of phototransduction in the retinal rod. In the dark, sodium, calcium and magnesium ions flow into the rod outer segment through channels held open by cyclic GMP (cGMP). Upon excitation by light, rhodopsin $(R h)$ in the disc membrane activates the G-protein transducin (T), which in turn activates cGMP phosphodiesterase (PDE). PDE hydrolyses CGMP, causing intracellular cGMP levels to drop and channels on the surface membrane to close. The decrease in the inward current hyperpolarises the rod, causing a reduced rate of neurotransmitter release from the synaptic terminal.

rhodopsin kinase under conditions of high calcium concentration. ${ }^{6.7}$ When the calcium level inside the rod drops during the response to light, inhibition of the kinase by recoverin should be removed, causing rhodopsin deactivation to proceed more rapidly, thus producing the decreased sensitivity characteristic of light adaptation. An alternative pathway for rhodopsin deactivation may be provided by a truncated splice variant of arrestin, p44, which can bind to unphosphorylated rhodopsin and strongly inhibit its activity. ${ }^{8}$ Thus, p44 may provide an important backup mechanism for phosphorylation-independent shut-off of rhodopsin. Failure of proper rhodopsin deactivation is presumably the cause of the functional defect in Oguchi disease, a recessively inherited form of stationary night blindness that is linked to homozygous deletions or missense mutations in the arrestin ${ }^{9}$ or rhodopsin kinase ${ }^{10}$ genes.

How is rhodopsin deactivated under physiological conditions? Do rhodopsin kinase and arrestin indeed function sequentially, as in the scheme of Fig. 2? What are the individual contributions of phosphorylation and arrestin binding to deactivation, and what happens if either process fails? Which site(s) on rhodopsin are phosphorylated? The purpose of this paper is to review recent physiological work that has examined these questions. The approach was to observe phototransduction in rods of transgenic mice in which the primary structure of rhodopsin was altered or one or both genes for arrestin was deleted. The molecular biology was performed in the laboratory of Professor Melvin Simon at the California Institute of Technology, using methods that have been described elsewhere. ${ }^{11,12}$ Immunoblot analysis was used to compare the levels of phototransduction proteins in transgenic and control animals. The expression of truncated rhodopsin and the absence of arrestin did not appear to affect the relative expression levels of other transduction proteins, although rearing the animals in the light caused shortening of the outer segments in some transgenics. Phototransduction in single rods was monitored by using a suction electrode to record the reduction in inward current that resulted from flashes of $500 \mathrm{~nm}$ light (Fig. 3). This method allows one to observe the rod's response to a single photon. ${ }^{13}$

\section{Results and discussion}

\section{Role of phosphorylation in deactivation of rhodopsin}

Previous studies demonstrated that supplying ATP to truncated, internally dialysed salamander rod outer segments reduced the amplitude and duration of the flash response, suggesting a role for phosphorylation in deactivation of the cascade. ${ }^{14}$ The C-terminus of rhodopsin contains multiple serine and threonine residues that can be phosphorylated after photoisomerisation occurs. Three of these sites (S334, S338, S343) have been implicated as the preferred sites for rhodopsin kinase. ${ }^{15}$ Pharmacological inhibition of rhodopsin kinase in isolated rod outer segments interfered with the recovery of light reponses, suggesting that phosphorylation of rhodopsin by rhodopsin kinase reduces its catalytic activity. ${ }^{4}$ To test whether phosphorylation of rhodopsin at its C-terminus is



Fig. 2. Rhodopsin deactivation scheme derived from biochemical experiments. After absorption of a photon $(h v)$, rhodopsin $(R h)$ is activated $\left(R h^{* *}\right)$ until it is phosphorylated by rhodopsin kinnse ( $R h$ kinase), which reduces its catalytic activity (Rh*). In the presence of calcium, rhodopsin kinase is inhibited by calcium-bound recoverin (Rec-Ca), which makes rhodopsin deactivation calcium-sensitive. Arrestin binds to phosphorylated rhodopsin $\left(R h_{n P}{ }^{*}\right)$, further que'nching its activity. A splice variant of arrestin ( $p 44)$ can bind to and inhibit the activity of unphosphorylated rhodopsin, providing an alternative deactivation pathway that is independent of phosphorylation. 




Fig. 3. Method for recording the light responses of a single mouse rod. Photomicrograph of a mouse rod outer segment in a small piece of retina, drawn into a suction electrode for recording the inward current in darkness (typically about $10 \mathrm{pA}$ ). Flashes of light decrease the dark current by closing cGMP-gated channels. By this method one can readily observe the cell's responses to absorption of single photons, which typically decrease the dark current by about $0.4 \mathrm{pA}$.

required for normal shut-off, a truncated rhodopsin gene (S334ter) was constructed (Fig. 4) and expressed in a background of normal rhodopsin gene expression at a ratio of about 1:10. Mutant S334ter lacked all the potential sites of phosphorylation by rhodopsin kinase.

Electrical recordings from rods expressing the truncated rhodopsin revealed greatly prolonged bright flash responses that eventually terminated in a series of prolonged steps. This finding was pursued by analysing responses to single photons, which are triggered by photoisomerisation of single rhodopsin molecules. In

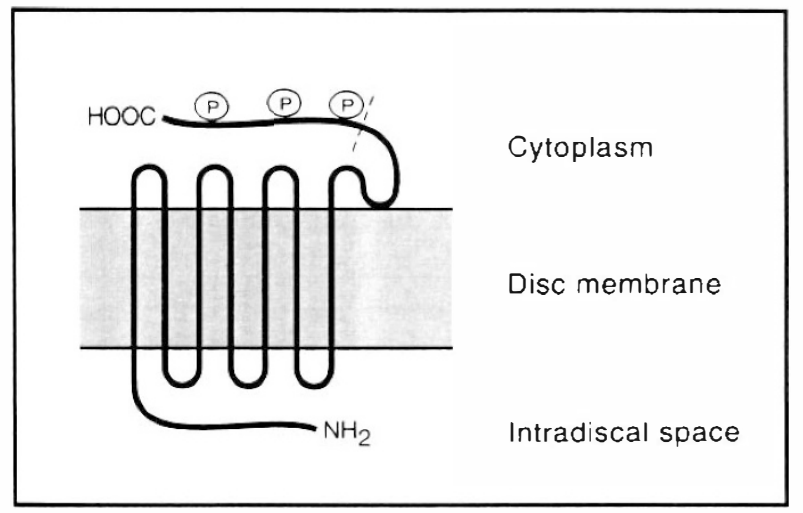

Fig. 4. Schematic diagram of rhodopsin. Rhodopsin, a member of the 7-transmembrane helix receptor family, resides in the disc membrane with its amino (N)-terminus in the intradiscal space and its carboxy (C)-terminus in the cytoplasm. The C-terminal domain contains the sites for phosphorylation by rhodopsin kinase, three of which are shown. The site of termination in the rhodopsin truncation mutant (S334ter) is indicated by the dashed line.
$10 \%$ of cases the single photon response was anomalous, with a rectangular waveform, an amplitude 2 times larger than normal, and a very prolonged time course. In $90 \%$ of the trials the single photon response was entirely normal. The relative numbers of normal and anomalous responses corresponded well with the measured expression ratio of wild-type and truncated rhodopsin in the outer segment, indicating that the anomalous responses resulted from the photoisomerisation of truncated rhodopsin molecules. This in turn supports the notion that phosphorylation at one or more sites at the Cterminus of rhodopsin is essential for normal deactivation. The fact that most of the single photon responses were normal provided evidence that the anomalous responses indeed resulted from a failure of truncated rhodopsin to deactivate properly, rather than from some other problem downstream in the cascade.

After a prolonged delay, the anomalous single photon responses abruptly recovered, resulting in a rectangular waveform (Fig. 5). The average duration of this delay was about 20 times the duration of the normal response. Furthermore, the delay varied widely: frequently the response would abruptly terminate after only a few seconds, while occasionally a response would last for over $10 \mathrm{~s}$. This is completely unlike the behaviour of the normal single photon response, which is remarkably reproducible. The frequency distribution of the duration of the anomalous responses followed an exponential curve, with a mean duration of about $5 \mathrm{~s}$, suggesting that a single first-order step was rate-limiting for termination of the anomalous responses.

The mechanism of this phosphorylation-independent shut-off is not known, although one possibility is that it results from the binding of $\mathrm{p} 44$ arrestin to unphosphorylated rhodopsin. ${ }^{8,16}$ Alternatively, there might be additional sites of phosphorylation - perhaps targets for another kinase - in a second pathway for deactivation. To distinguish between these possibilities, it will be interesting to examine rods that lack rhodopsin kinase or overexpress p44. If rhodopsin kinase is solely responsible for rhodopsin phosphorylation in vivo, the dim flash responses in rods lacking rhodopsin kinase should resemble those of the rhodopsin truncation mutant. If shut-off of S334ter rhodopsin results from the binding of $\mathrm{p} 44$, then overexpressing p44 should increase the rate of deactivation and shorten the mean duration of the anomalous responses.

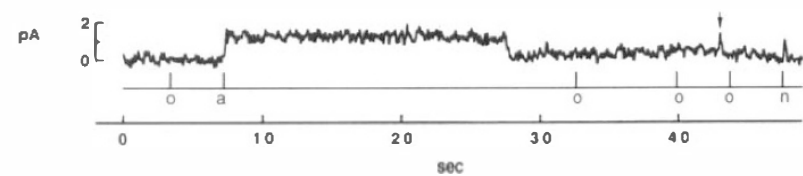

Fig. 5. Flash responses of a rod expressing S334ter rhodopsin. Brief flashes of dim $500 \mathrm{~nm}$ light (4.4 photons $/ \mu^{2}{ }^{2}$ ) were delivered to the rod at the times indicated by the lines in the flash monitor trace below the record. Several flashes (o) elicted no responses. One flash (a) caused an anomalous single photon response lasting approximately $20 \mathrm{~s}$, while another flash $(n)$ elicited a normal single photon response. A noise event resulting from a spontaneous thermal isomerisation of rhodopsin is marked by the arrow. 
Another question concerns the role of the multiple sites for phosphorylation at rhodopsin's C-terminus: Is phosphorylation at one site sufficient or are all sites necessary for proper deactivation? Do the sites phosphorylated change when recoverin-calcium inhibits phosphorylation? Single amino acid substitutions of rhodopsin's phosphorylation sites and knock-out of recoverin expression should help to answer these questions.

Curiously, normal monkey rods occasionally exhibit anomalous elementary responses very similar to those generated by truncated rhodopsin. ${ }^{17}$ In response to bright flashes, the monkey rods also display prolonged responses resulting from the superimposition of multiple


Fig. 6. Failure of normal recovery of responses from arrestin-deficient rods. (A) Averaged responses from a control rod $(A+1+)$ and a rod from an arrestin knock-out mouse $(A-/-)$ in response to flashes of increasing strength $\left(8.3,53.7,99.4,361\right.$ and 1210 photons $/ \mu m^{2}$ at 500 $\mathrm{nm}$ for the $A+1+\operatorname{rod}$ and 20.9, 76, 133, 431 and 3010 photons/ $\mathrm{\mu m}^{2}$ for the A-I- rod). Responses were normalised by the saturating response amplitudes. Flashes were delivered at the time indicated by the arrow. (B) Average of $63 \mathrm{dim}$ flash responses from a $A-1-$ rod shown on an expanded time scale. Flashes were delivered at time zero on abscissa. Reprinted by permission of Macmillan Publishers Ltd from $X u$ et al. ${ }^{12}$ steps. The similarity of these anomalous single photon responses to those triggered by S334ter rhodopsin suggests that in normal rods roughly one rhodopsin molecule in a thousand may be proteolysed or fail to be synthesised fully in vivo. These defective rhodopsin molecules could perhaps account for the first component of the threshold elevation that follows bright light exposure in psychophysical experiments. ${ }^{18}$ This component of human dark-adaptation has a time constant of about $5 \mathrm{~s},{ }^{19,20}$ consistent with the time course of the anomalous photon responses.

\section{Role of arrestin}

The observations on the rods expressing truncated rhodopsin indicated that phosphorylation is required to initiate normal rhodopsin deactivation and recovery of the photoresponse. But is phosphorylation itself sufficient to inactivate rhodopsin? In an attempt to answer this question, one or both genes for arrestin (and thus also its splice variant, p44) were deleted.

Hemizygous rods possessing one copy of the arrestin gene and expressing only about half the normal amount of arrestin had normal flash responses. This indicates that arrestin is normally present in excess in the outer segment. Since the rate of arrestin binding to an activated rhodopsin should be directly proportional to arrestin concentration, halving the amount of arrestin should halve the rate of arrestin binding. If arrestin binding to rhodopsin were the rate-limiting step in recovery of the normal rod response, recovery in the hemizygous rods should be slowed. As this was not observed, arrestin binding is apparently not rate-limiting for final recovery in normal rods, unlike the situation in Drosophila photoreceptors. $^{21,22}$

Rods lacking both copies of the arrestin gene had very unusual photoresponses. The flash responses rose and began to recover normally, but the final stage of recovery was greatly slowed, with a time constant of about $40 \mathrm{~s}$ (Fig. 6). Thus, arrestin is essential for complete and rapid deactivation in vivo. Although the mechanism for the gradual deactivation in the arrestin knock-out is not known, the time course is similar to that of the thermal decay of metarhodopsin II. This suggests that no alternative mechanism for final deactivation can substitute for arrestin binding.

The flash responses of the transgenic rods are thus consistent with the notion that shut-off of rhodopsin's catalytic activity is initiated by phosphorylation and completed by arrestin binding. With defective phosphorylation (S334ter), the single photon response continued to rise for longer than normal and reached a plateau that was maintained for seconds at an amplitude twice that of the normal response. With arrestin absent $(\mathrm{A}-/-)$, the response stopped rising at the appropriate time and began to recover, although final recovery was very slow. The difference between the responses of S334ter and $\mathrm{A}-/$ - is attributable to the effect of phosphorylation alone. The 2-fold difference in the response amplitudes suggests that phosphorylation 
reduces rhodopsin's catalytic activity at least 2 -fold. Comparison of responses from rhodopsin kinase knockout rods with those from S334ter may make it possible to determine the respective contributions of kinase binding and phosphorylation to the fast initial phase of rhodopsin deactivation.

The functional defects observed in the transgenic mouse rods give important clues about the defects in Oguchi disease. The stationary night blindness in these patients probably results at least in part from abnormally prolonged single photon responses, which, at any given background light level, will disproportionately desensitise the rod system. A more puzzling observation is that in patients with Oguchi disease dark adaptation is greatly prolonged, requiring up to $4 \mathrm{~h} .{ }^{10}$ We can speculate that free opsin, if not phosphorylated and bound to arrestin, has residual catalytic activity, and that therefore even a few molecules of unregenerated pigment have a larger than normal desensitising effect on the rod system. This explanation provides a specific mechanism for the somewhat similar suggestion put forward by Yamamoto et al. ${ }^{10}$

To summarise, the deactivation of rhodopsin appears to have multiple stages and back-up mechanisms, as one might expect for a process that is fundamental to good night vision. Both rhodopsin phosphorylation and arrestin binding are required for normal response recovery and reproducibility; however, perturbation of one or the other does not result in complete failure of recovery, only failure of normal recovery. Perhaps the subtlety of the functional deficits explains why patients with homozygous defects in rhodopsin kinase or arrestin suffer from a stationary night blindness rather than retinitis pigmentosa.

Drs Clint Makino and Robert Dodd made the electrical recordings described here, and the molecular biology was performed by Drs Jeannie Chen and Jun Xu in the laboratory of Dr Melvin Simon

\section{References}

1. Wilden $\mathrm{U}, \mathrm{Kühn} \mathrm{H}$. Light-dependent phosphorylation of rhodopsin: number of phosphorylation sites. Biochemistry 1982;21:3014-22.

2. Palczewski K, McDowell JH, Hargrave PA. Purification and characterization of rhodopsin kinase. J Biol Chem 1988;263:14067-73.

3. Wilden U, Hall SW, Kühn H. Phosphodiesterase activation by photoexcited rhodopsin is quenched when rhodopsin is phosphorylated and binds the intrinsic 48-kDa protein of rod outer segments. Proc Natl Acad Sci USA 1986;83:1174-8.
4. Palczewski K, Rispoli G, Detwiler PB. The influence of arrestin (48K protein) and rhodopsin kinase on visual transduction. Neuron 1992;8:117-26.

5. Kawamura S. Rhodopsin phosphorylation as a mechanism of cyclic GMP phosphodiesterase regulation by S-modulin. Nature 1993;362:855-7.

6. Calvert PD, Klenchin VA, Bownds MD. Rhodopsin kinase inhibition by recoverin: function of recoverin myristoylation. J Biol Chem 1995;270:24127-9.

7. Chen CK, Inglese J, Lefkowitz RJ, Hurley JB. $\mathrm{Ca}^{(2+)}$ dependent interaction of recoverin with rhodopsin kinase. J Biol Chem 1995;270:18060-6.

8. Smith WC, Milam AH, Dugger D, Arendt A, Hargrave PA, Palczewski K. A splice variant of arrestin: molecular cloning and localization in bovine retina. J Biol Chem 1994;269:15407-10.

9. Fuchs S, Nakazawa M, Maw M, Tamai M, Oguchi Y, Gal A. A homozygous 1-base pair deletion in the arrestin gene is a frequent cause of Oguchi disease in Japanese. Nature Genet 1995;10:360-2.

10. Yamamoto S, Sippel KC, Berson EL, Dryja TP. Defects in the rhodopsin kinase gene in the Oguchi form of stationary night blindness. Nature Genet 1997;15:175-8.

11. Chen J, Makino CL, Peachey NS, Baylor DA, Simon MI. Mechanisms of rhodopsin inactivation in vivo as revealed by a COOH-terminal truncation mutant. Science 1995;267:374-7.

12. Xu J, Dodd RL, Makino CL, Simon MI, Baylor DA, Chen J. Prolonged photoresponses in transgenic mouse rods lacking arrestin. Nature 1997;389:505-9.

13. Baylor DA, Lamb TD, Yau K-W. Responses of retinal rods to single photons. J Physiol (Lond) 1979;288:613-34.

14. Yau K-W, Nakatani K. Light-suppressible, cyclic GMPsensitive conductance in the plasma membrane of a truncated rod outer segment. Nature 1985;317:252-5.

15. Ohguro H, Rudnicka-Nawrot M, Buczylko J, Zhao X, Taylor JA, Walsh KA, Palczewski K. Structural and enzymatic aspects of rhodopsin phosphorylation. J Biol Chem 1996;271:5215-24.

16. Pulvermüller A, Maretzki D, Rudnicka-Nawrot M, Smith WC, Palczewski K, Hofmann KP. Functional differences in the interaction of arrestin and its splice variant, p44, with rhodopsin. Biochemistry 1997;36:9253-60.

17. Baylor DA, Nunn BJ, Schnapf JL. The photocurrent, noise, and spectral sensitivity of rods of the monkey Macaca fascicularis. J Physiol (Lond) 1984;357:575-607.

18. Barlow HB. Dark and light adaptation: psychophysics. In: Jameson D, Hurvich LM, editors. Handbook of sensory physiology, vol VII/4. Visual psychophysics. New York: Springer, 1972:1-28.

19. Pugh EN. Rushton's paradox: rod dark adaptation after flash photolysis. J Physiol (Lond) 1975;248:413-31.

20. Lamb TD. The involvement of rod photoreceptors in dark adaptation. Vision Res 1981;21:1773-82.

21. Dolph PJ, Ranganathan R, Colley NJ, Hardy RW, Socolich M, Zuker CS. Arrestin function in inactivation of $G$ proteincoupled receptor rhodopsin in vivo. Science 1993;260:1910-6.

22. Ranganathan R, Stevens CF. Arrestin binding determines the rate of inactivation of the $G$ protein-coupled receptor rhodopsin in vivo. Cell 1995;81:841-8. 